\title{
Static Aeroelastic Response of Wing-Structures Accounting for In-Plane Cross-Section Deformation
}

\author{
Alberto Varello* and Alessandro Lamberti** \\ Department of Mechanical and Aerospace Engineering, Politecnico di Torino, 10129 Torino, Italy \\ Erasmo Carrera*** \\ Department of Mechanical and Aerospace Engineering, Politecnico di Torino, 10129 Torino, Italy \\ Faculty of Science, King Abdulaziz University, Jeddah 21589, Saudi Arabia
}

\begin{abstract}
In this paper, the aeroelastic static response of flexible wings with arbitrary cross-section geometry via a coupled CUFXFLR5 approach is presented. Refined structural one-dimensional (1D) models, with a variable order of expansion for the displacement field, are developed on the basis of the Carrera Unified Formulation (CUF), taking into account cross-sectional deformability. A three-dimensional (3D) Panel Method is employed for the aerodynamic analysis, providing more accuracy with respect to the Vortex Lattice Method (VLM). A straight wing with an airfoil cross-section is modeled as a clamped beam, by means of the finite element method (FEM). Numerical results present the variation of wing aerodynamic parameters, and the equilibrium aeroelastic response is evaluated in terms of displacements and in-plane cross-section deformation. Aeroelastic coupled analyses are based on an iterative procedure, as well as a linear coupling approach for different free stream velocities. A convergent trend of displacements and aerodynamic coefficients is achieved as the structural model accuracy increases. Comparisons with 3D finite element solutions prove that an accurate description of the in-plane crosssection deformation is provided by the proposed $1 \mathrm{D}$ CUF model, through a significant reduction in computational cost.
\end{abstract}

Key words: aeroelasticity, higher-order 1D finite elements, Carrera Unified Formulation, in-plane cross-section deformation

\section{Introduction}

The in-depth understanding of aeroelastic effects on deformable lifting bodies (LBs), due to steady and unsteady aerodynamic loadings, is a typical challenging issue for the current design of aerospace vehicles [1]. Furthermore, with the forthcoming employment of composite materials in nextgeneration aircraft configurations, such as High-Altitude Long Endurance aircraft (HALE) [2], and strut-braced wings [3], accurate evaluation of the aeroelastic response becomes even more crucial [4].

Recently, special attention has been directed to the profitable exploitation of the aeroelastic phenomena comprehension, by studying the concept of morphing wings, which are able to adapt and optimize their shape depending on the specific flight conditions and mission profiles $[5,6]$. The smart wing is very flexible and could allow a number of advantages, such as drag reduction and aeroelastic vibrations suppression by means of adaptive control [7] and different solutions, such as compliant structures [8], bi-stable laminate composites [9], piezoelectric [10] and shape memory alloy actuation [11].

In order to develop aeroelastic tools that are able to work in any regime and with any LB geometry, the literature from the last decades has been widely influenced by research devoted to build reliable methods, to couple computational fluid dynamics (CFD) or classical aerodynamic methods with the finite element method (FEM) for structural modeling [12].
This is an Open Access article distributed under the terms of the Creative Commons Attribution Non-Commercial License (http://creativecommons.org/licenses/by$\mathrm{nc} / 3.0 /$ which permits unrestricted non-commercial use, distribution, and reproduction in any medium, provided the original work is properly cited. cc * Research assistant, corresponding author: alberto.varello@polito.it ** Ph.D. student

*** Professor of Aerospace Structures and Aeroelasticity 
Valuable examples are in the review articles by Dowell and Hall [13], and Henshaw et al. [14]. Reduced approaches, for instance panel methods, are widely used for the classical aerodynamics of wings under some limitations [15], allowing a sizeable reduction in terms of computational cost $[16,17]$. The assumption of undeformable airfoil-crosssections [18] is typically not proper for recent configurations, since the weight reduction makes wings more flexible and highly-deformable. Hence, two-dimensional (2D) plate/ shell and three-dimensional (3D) solid methods are usually employed for the structural modeling, instead of classical one-dimensional (1D) theories, such as the Euler-Bernoulli, Timoshenko, or Vlasov theories [19].

With the advent of smart wings, detailed structural and aeroelastic models are even more essential to fully exploit the non-classical effects in wing design, due to the properties characterizing advanced composite materials, such as anisotropy, heterogeneity and transverse shear flexibility. Beam-like components can be analyzed by means of refined one-dimensional (1D) formulations, which have the main advantage of a lower computational cost required compared with 2D and 3D models. A detailed review of the recent development of refined beam models can be found in [20]. El Fatmi [21] improved the displacement field over the beam cross-section, by introducing a warping function, to refine the description of normal and shear stress of the beam. Generalized beam theories (GBT) originated with Schardt's work [22] and improved classical theories, by using a piecewise beam description of thin-walled sections [23]. An asymptotic type expansion, in conjunction with variational methods, was proposed by Berdichevsky et al. [24], where a commendable review of prior works on beam theory development was given. An alternative approach to formulating refined beam theories, based on asymptotic variational methods (VABS), has led to an extensive contribution in the last few years [25].

A considerable amount of research activity devoted to aeroelastic analysis and optimization was undertaken in the last decades, by using reduced $1 \mathrm{D}$ models. A review was carried out by Patil [26], who investigated the variation of aeroelastic critical speeds with the composite ply layup of box beams, via the unsteady Theodorsen's theory. A thin-walled anisotropic beam model in-corporating nonclassical effects was introduced by Librescu and Song [27] to analyze the sub-critical static aeroelastic response, and the divergence instability of swept-forward wing structures. Qin and Librescu [28] developed an aeroelastic model to investigate the influence of the directionality property of composite materials, and non-classical effects on the aeroelastic instability of thin-walled aircraft wings. Among the several composite rotor blades applications, the work done by Jeon and Lee [29] concerning the steady equilibrium deflections, via a large deflection type beam theory with small strains, is worth mentioning. An example of the use of a refined beam theory for aeroelastic analysis can be found in [30], where the static and dynamic responses of a helicopter rotor blade are evaluated by means of a YF/VABS model.

Higher-order 1D models with generalized displacement variables, based on the Carrera Unified Formulation, have recently been proposed by Carrera and co-authors, for the static and dynamic analysis of isotropic and composite structures [31]. The CUF is a hierarchical formulation, which considers the order of the model as a free-parameter of the analysis. In other words, models of any order can be obtained, with no need for ad hoc formulations, by exploiting a systematic procedure. Structural 1D CUF models were used to analyze the structural response of isotropic aircraft wings, under aerodynamic loads computed through the Vortex Lattice Method (VLM), in [32]. The aeroelastic CUF-VLM coupling was preliminarily formulated in [33] for isotropic flat plates, and then extended to instability divergence detection and the evaluation of composite material lay-up effects on the aeroelastic response of moderate and high-aspect ratio wing configurations, in [34]. Flutter analyses of composite lifting surfaces were also presented in [35], by coupling the CUF approach with the Doublet Lattice Method.

The present work couples a refined one-dimensional finite element model based on CUF to an aerodynamic 3D Panel Method, implemented in the software XFLR5. Two potential methods are here compared: the VLM and the 3D Panel Method. The aeroelastic static response of a straight wing is computed through a coupled iterative procedure, and a linear coupling approach. Particular attention is drawn to the in-plane deformation of the wing airfoil cross-sections, as well as the aeroelastic influence of free stream velocity.

\section{Numerical models: refined 1D CUF model and panel methods}

\subsection{Variable kinematic 1D CUF FE model}

For the sake of completeness, some details about the formulation of CUF finite elements are here retrieved from previous works [32, 34]. A structure with axial length $L$ and cross-section $\Omega$ is discretized through a mesh of $N_{E L} 1 \mathrm{D}$ finite elements. A cartesian coordinate system is defined with axes $x$ and $z$ parallel to the cross-section, whereas $y$ represents the longitudinal coordinate. According to the displacementbased framework of CUF [31], the displacement field is 
assumed to be an expansion of a certain class of functions $F_{\tau}$, which depend on the cross-section coordinates $x$ and $z$. Introducing the shape functions $N_{i}$ and the nodal displacement vector $\boldsymbol{q}$, the displacement vector $\boldsymbol{u}$ with components $u_{x}, u_{y}$, and $u_{z}$ becomes:

$$
\begin{aligned}
& \boldsymbol{u}(x, y, z)=F_{\tau}(\mathrm{x}, \mathrm{z}) N_{i}(y) \boldsymbol{q}_{\tau i} \\
& i=1, \ldots, N_{N} \\
& \tau=1, \ldots,(N+1)(N+2) / 2
\end{aligned}
$$

where $\boldsymbol{q}_{\tau i}$ contains the degrees of freedom of the $\tau$-th expansion term corresponding to the $i$-th element node. The compact expression in Eq. 1 is based on Einstein's notation: repeated subscripts $\tau$ and $i$ indicate summation. Multivariate Taylor's polynomials of the $x$ and $z$ variables are employed here as cross-section functions $F_{\tau}$, and $N$ is defined as the expansion order, which is a free parameter of the formulation. Elements with number of nodes $N_{N}=4$ are formulated in the present work, and named B4, using third-order Lagrange polynomials as shape functions [19]. The number of degrees of freedom (DOFs) used through the proposed approach is:

$$
\text { DOFs }=3 \frac{(N+1)(N+2)}{2}\left(3 N_{E L}+1\right)
$$

The variational statement employed is the Principle of Virtual Displacements:

$$
\delta L_{i n t}=\int_{V}\left(\delta \boldsymbol{\varepsilon}_{n}^{T} \boldsymbol{\sigma}_{n}+\delta \boldsymbol{\varepsilon}_{p}^{T} \boldsymbol{\sigma}_{p}\right) d V=\delta L_{e x t}=\delta \boldsymbol{u}^{T} \mathbf{P}
$$

where $L_{i n t}$ is the internal strain energy, and $L_{e x t}$ is the work of external loadings variationally consistent with the present method, and here derived for the case of a generic concentrated load $\boldsymbol{P}=\left\{P_{u_{x}} P_{u_{y}} P_{u_{z}}\right\}^{T}$, acting on the arbitrary load application point $\left(x_{P}, y_{P}, z_{P}\right)$, which does not necessarily lie along the 1D finite element mesh, unlike standard 1D FE models. $\delta$ stands for the virtual variation. By using Eq. 1, $\delta L_{\text {ext }}$ becomes:

$$
\delta L_{e x t}=\delta \boldsymbol{u}^{T} \mathbf{P}=\delta \boldsymbol{q}_{\tau i}^{T} F_{\tau} N_{i} \mathbf{P}=\delta \boldsymbol{q}_{\tau i}^{T} \boldsymbol{F}_{\tau i}
$$

where $F_{\tau}$ is evaluated in $\left(x_{P}, z_{P}\right)$ and $N_{i}$ is calculated in $y_{P}$. In the case of small displacements with respect to the length $L$, the in-plane (subscript $p$ ) and out-of-plane (subscript $n$ ) cross-section stress and strain vectors in Eq. 3 are related to the displacement vector via linear differential matrix operators $\boldsymbol{D}_{p}, \boldsymbol{D}_{n}$, and material stiffness matrices $\boldsymbol{C}_{p p}, \boldsymbol{C}_{p n}$, $\boldsymbol{C}_{n p}, \boldsymbol{C}_{n n}$, as follows:

$$
\begin{array}{ll}
\boldsymbol{\varepsilon}_{p}=\boldsymbol{D}_{p} \boldsymbol{u} & \boldsymbol{\sigma}_{p}=\boldsymbol{C}_{p p} \boldsymbol{\varepsilon}_{p}+\boldsymbol{C}_{p n} \boldsymbol{\varepsilon}_{n} \\
\boldsymbol{\varepsilon}_{n}=\boldsymbol{D}_{n} \boldsymbol{u} & \boldsymbol{\sigma}_{n}=\boldsymbol{C}_{n p} \boldsymbol{\varepsilon}_{p}+\boldsymbol{C}_{n n} \boldsymbol{\varepsilon}_{n}
\end{array}
$$

Using Eq. 5, Eq. 3 can be rewritten in terms of virtual nodal displacements:

$$
\delta \boldsymbol{q}_{\tau i}^{T} \boldsymbol{K}^{i j \tau s} \boldsymbol{q}_{s j}=\delta \boldsymbol{q}_{\tau i}^{T} \boldsymbol{F}_{\tau i}
$$

where, the $3 \times 3$ and $3 \times 1$ fundamental nuclei $\boldsymbol{K}^{i j \tau s}$ and $\boldsymbol{F}_{\tau i}$ are introduced. From Eq. 6 , the governing equation of motion can be derived through a finite element assembly procedure:

$$
\boldsymbol{K} \boldsymbol{q}=\boldsymbol{F}
$$

where $\boldsymbol{K}$ is the structural stiffness matrix, and $\boldsymbol{F}$ is the vector of equivalent nodal forces. It should be noted that no assumptions on the expansion order have so far been made. Therefore, it is possible to obtain higher-order 1D models without changing the formal expression of the nuclei components, as well as classical beam models, such as Euler-Bernoulli's and Timoshenko's. Higher-order models provide an accurate description of the shear mechanics, the in-plane and out-of-plane cross-section deformation, Poisson's effect along the spatial directions, and the torsional mechanics, in more detail than classical models do. Thanks to the CUF, the present hierarchical approach is invariant with respect to the order of the displacement field expansion. More details are not reported here, but can be found in the work of [31].

\subsection{A numerical approach for wing aerodynamic analysis}

\subsubsection{Preliminaries}

The evaluation of aerodynamic loads can be typically carried out through a CFD code, which solves, for example, either Navier-Stokes equations or Euler equations numerically. This kind of analysis has a high computational cost, but under some assumptions it is possible to employ simplified approaches. In the wing cases considered in the present work, the flow field is assumed to be steady, and the fluid viscosity is not decisive since the viscous effects can be confined into a small region (boundary layers and wake regions). The fluid can be thus considered as inviscid, and the flow field is irrotational, since the curl of the velocity vector $\boldsymbol{V}(x, y, z)$ is equal to zero:

$$
\nabla \times \boldsymbol{V}=\mathbf{0}
$$

In this case, the velocity vector $\boldsymbol{V}(x, y, z)$ can be considered as the gradient of a potential function $\phi$ :

$$
\nabla \phi=V
$$

Hence, the analysis of a wing or an airfoil under these conjectures can be performed by potential methods. The potential function describing the flow field around an object 
can be defined as a combination of singularities, such as doublets, vortices, sources, or uniform flux over the external body surface. According to the detailed exposition in [36], the equation to be used to compute the solution of the aerodynamic problem is Laplace's equation:

$$
\nabla^{2} \phi=0
$$

Laplace's equation describes a potential flow field only if the compressibility effects can be neglected, as occurs for the results presented afterwards, where the free stream velocity is rather low. Otherwise, some corrections, e.g. the Prandtl-Glauert transformation, are necessary, as explained in [37]. The assumptions here introduced lead to an integraldifferential equation, which expresses the potential function in an arbitrary point of the fluid domain as a combination of singularities. For the sake of brevity, this equation is not reported here, but more details can be found in [36]. Among the potential methods, the panel methods can be formulated following a low-order, or a high-order approach. The loworder (first-order) panel method employs triangular or quadrilateral panels having constant values of singularities' strength, such as the Hess and Smith approach. The higherorder panel methods instead use higher than first-order panels (e.g. paraboloidal panels) and a varying singularity strength over each panel.

\subsubsection{XFLR5: an implementation of aerodynamic potential methods}

XFLR5 is a software developed by Andre Deperrois. It performs viscous and inviscid aerodynamic analysis on airfoils and wings, using three potential methods: the Lifting Line Theory (LLT), the VLM, and the 3D Panel Method. The LLT method derives from Prandtl's wing theory and considers the wing as a linear distribution of vortices. The VLM considers a wing as an infinitely thin lifting surface, via a distribution of vortices placed over a wing reference surface. This method requires the non-penetration condition on the reference surface as a boundary condition. Hence, the normal component of the induced velocity $\boldsymbol{V}_{i}$ on the generic $\boldsymbol{i}$-th aerodynamic panel with normal vector $\boldsymbol{n}_{i}$ is equal to zero:

$$
\boldsymbol{V}_{i} \cdot \boldsymbol{n}_{i}=0
$$

Further details on this method can be found in [15]. The 3D Panel Method schematizes the wing surface as a distribution of doublets and sources. The strength of the doublets and sources is calculated to meet the appropriate boundary conditions (BCs), which may be of Dirichlet- or Neumann-type. According to the creator of the program, after a trial and error process, the best results can be obtained by using just the Dirichlet BC type [38]. The 3D Panel Method employs a low-order panel method. The LLT approach is not able to evaluate the pressure coefficients on the wing surface, but only the lifting loads along the lifting line. The VLM is able to analyze the pressure coefficients, but only on the reference surface, which is defined as the mean surface between the upper and the lower wing surfaces. The 3D Panel Method is able to calculate the pressure coefficients on both the upper and the lower wing surfaces. Therefore, this method offers the most realistic description of the aerodynamic field.

\section{Aeroelastic static response analysis via the CUF-XFLR5 approach}

In this work the aeroelastic static response of the wing is computed through an iterative procedure, based on a coupled CUF-XFLR5 method. Hence, the aerodynamic analysis is performed through the potential methods available in XFLR5, as previously mentioned; whereas, variable kinematic 1D CUF models provide the structural wing deformation with a variable expansion $\operatorname{order} N$.

\subsection{Iterative procedure}

Figure 1 shows in detail the aeroelastic iterative process, which starts with the evaluation of the pressure coefficients for the undeformed wing configuration. The further steps to be repeated for each iteration are:

1. post-processing calculation of the aerodynamic forces;

2. structural analysis of the wing, subject to the aerodynamic forces previously computed;

3. new calculation of the aerodynamic pressure coefficients for the new deformed configuration;

4. post-processing evaluation of the wing deformation and cross-section distortion.

Structural displacements are evaluated in specific sections distributed regularly along the wing span. The cross-section distortion $s$ is defined as the in-plane displacement, i.e. a quantity that expresses the in-plane difference between the deformed shape and the "undeformed" shape of the airfoil cross-section:

$$
s=\sqrt{\Delta u_{x}^{2}+\Delta u_{z}^{2}}
$$

where $\Delta u_{x}$ and $\Delta u_{z}$ are the cartesian components of the relative displacement vector $\Delta \boldsymbol{u}$ along the chord direction $x$ and the transversal direction $z$, respectively, between 
the deformed cross-section and the base section. Given a structural model, the base section corresponds to the undeformed cross-section, shifted and rotated in such a way that its leading edge and trailing edge points correspond to the leading edge and trailing edge points, respectively, of the deformed cross-section obtained by such a structural model.

The iterative process in Fig. 1 is stopped once the convergences of the lifting coefficient $C_{L}$, the moment coefficient $C_{M}$, and the cross-section distortion of the wing sections are achieved simultaneously. The description of a similar iterative process can also be found in [12]. The convergence controls are thus:

$$
\begin{aligned}
& \frac{\left|C_{L}^{i}-C_{L}^{i-1}\right|}{C_{L}^{i}}<\text { toll } ; \frac{\left|C_{M}^{i}-C_{M}^{i-1}\right|}{C_{M}^{i}}<\text { toll } \\
& \frac{\left|\bar{s}^{i}-\bar{s}^{i-1}\right|}{\bar{s}^{i}}<\text { toll }
\end{aligned}
$$

where toll is equal to $10^{-4}, C_{L}^{i}, C_{M}^{i}$, and $\bar{s}^{i}$ are the lifting coefficient, the moment coefficient, and the average crosssection distortion for the generic i-th iteration, respectively. The average distortion $\bar{s}$ is defined in Eq. 18. A linear approach is adopted as usual in classical aeroelasticity: for each iteration, the aerodynamic loads computed for the deformed wing configuration are applied to the undeformed configuration, without changing the structural stiffness matrix $\boldsymbol{K}$ of Eq. 7.

\subsection{Aerodynamic loads computation}

The aerodynamic load computed by XFLR5 is a distributed pressure, and in this work it is modeled as distributed forces. The generic force acting on the $\mathrm{j}$-th aerodynamic panel is evaluated as:

$$
F_{j}=\frac{1}{2} \cdot \rho_{\infty} \cdot V_{\infty}^{2} \cdot A_{j} \cdot C_{p}^{j}
$$

where $V_{\infty}$ is the free stream velocity, and $\rho_{\infty}$ is the air density. $A_{j}$ is the area of the $\mathrm{j}$-th aerodynamic panel, which the pressure coefficient $C_{P}^{j}$ refers to. According to XFLR5 notation, normal vectors are considered positive when $C_{P}^{j}$ is negative, and their verse is outer-pointing. Each aerodynamic force is transferred from the aerodynamic model to the structural model, following the approach described in section 2.1 for the generic concentrated load $\boldsymbol{P}$.

For each iteration, the three-dimensional deformed configuration of the wing is built using 11 airfoils along the half-wing span, at a distance of $0.5 \mathrm{~m}$ from each other. The first section lies at the wing root. The wing is discretized through a lattice of quadrilateral aerodynamic panels. Let $N_{A P}^{x}$ be the number of panels along the chord line, and let $N_{A P}^{y}$ be the number of panels along the half-wing span. When the VLM is employed, the total number of aerodynamic panels $N_{A P}$ is equal to $2 N_{A P}^{x} N_{A P}^{y}$. For the 3D Panel Method, $N_{A P}$ must be calculated as $4 N_{A P}^{x} N_{A P}^{y}+2 N_{A P}^{x}$, where the term $4 N_{A P}^{x} N_{A P}^{y}$ is the number of panels along the wing span, on the upper and lower surfaces of the wing. In addition, the term $2 N_{A P}^{x}$ is the number of panels placed on the tip lateral crosssections. For the sake of convenience, only the half-wing is analyzed, since the aerodynamic loads are considered to be symmetric with respect to the wing root.

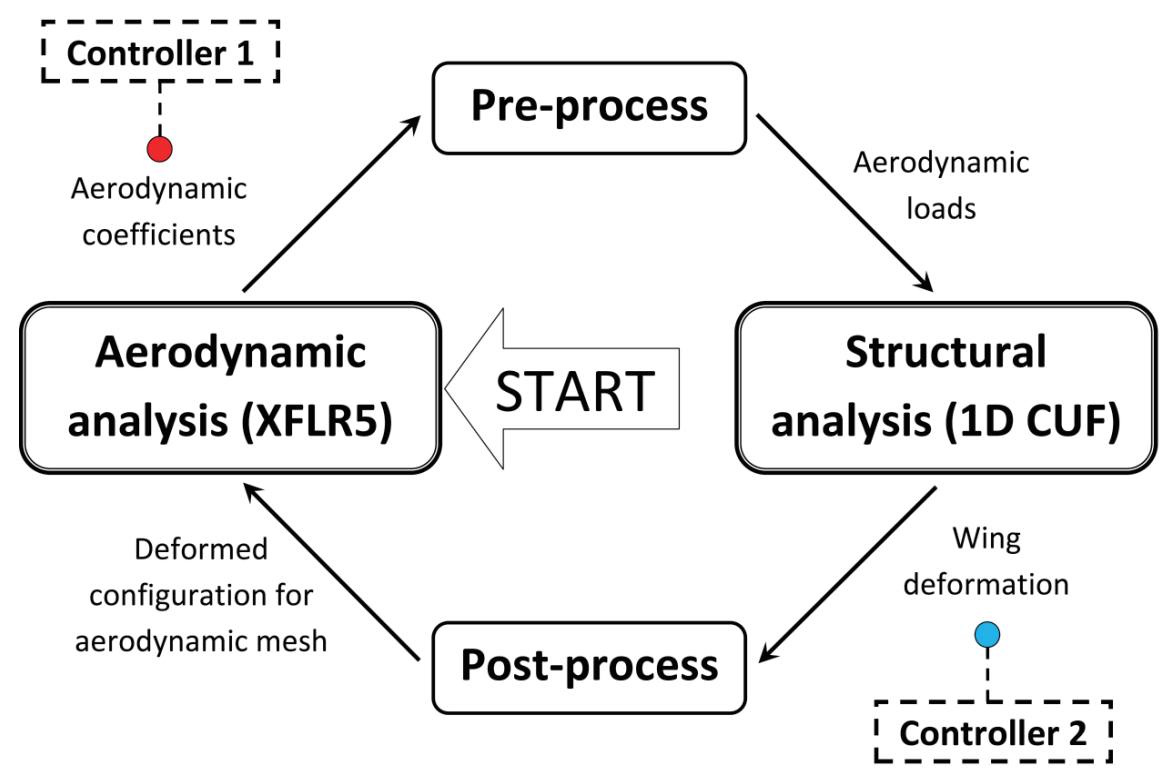

Fig. 1. Aeroelastic iterative procedure, with controllers on aerodynamic coefficients and wing deformation. 


\section{Numerical results}

\subsection{Aerodynamic assessment}

Firstly, an aerodynamic assessment of the VLM and the 3D Panel Method, which are able to evaluate the pressure coefficients on the wing surface, is performed analyzing the effects of two typical geometrical parameters: the airfoil thickness and the camber line. A straight wing is considered: the wing span is $10 \mathrm{~m}$, and the airfoil chord is $1 \mathrm{~m}$ long, as drawn in Fig. 2a, where the right half-wing is depicted. This wing configuration is also used in the following structural and aeroelastic analyses. The effect of the camber line on the aerodynamic field is evaluated, using NACA 2415, 3415 and 4415 airfoils. The analysis of the influence of the airfoil thickness is then carried out, using the symmetric NACA 0005, 0010 and 0015 airfoils. The number of aerodynamic panels is chosen as a compromise between the limit number of panels that can be used in XFLR5 $(=5,000)[38]$, and the number of panels required, in order to achieve convergence in the aerodynamic results. In the following analyses, the choice of $N_{A P}^{x}=24$ and $N_{A P}^{y}=50$ remains the same.

For the presentassessmentanalysis, thefree streamvelocity is assumed to be $V_{\infty}=50 \mathrm{~m} / \mathrm{s}$, such that the compressibility effects can be neglected. The air density is assumed to be $\rho_{\infty}$ $=1.225 \mathrm{~kg} / \mathrm{m}^{3}$. The angle of attack $\alpha$ of the wing is equal to 3 deg. In all the following analyses, the air density $\rho_{\infty}$ and the angle of attack $\alpha$ will be invariable parameters. The results focus on the variation of the spanwise local lifting coefficient $C_{l}$ along the wing span, defined as:

$$
C_{l}(y)=\frac{L(y)}{\frac{1}{2} \cdot \rho_{\infty} \cdot V_{\infty}^{2} \cdot 2 \cdot e(y) \cdot c(y)}
$$

where, $c(y)$ and $L(y)$ are the chord and the Lift Force, respectively, generated by the pressure acting on the panels with span-length $2 e(y)$, placed at the $y$ coordinate. More details can be found in [32]. As a first result, the trend of $C_{l}$ along the y axis (right half-wing) is reported in Fig. 3a. This analysis is carried out, considering the variation of the airfoil thickness. As expected, the VLM is not able to take into account the variation of airfoil thickness, since it computes

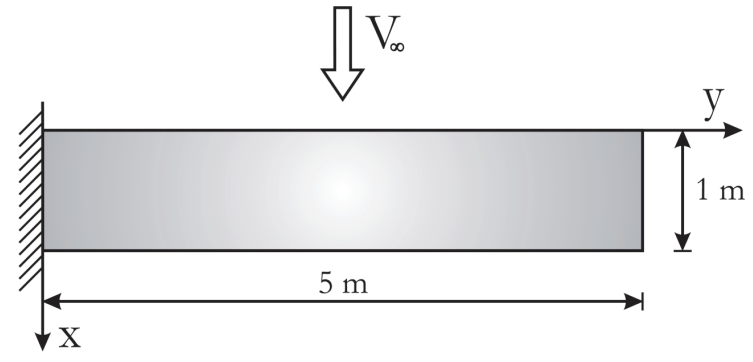

(a) Plan view of the straight half-wing

Fig. 2. Geometrical configuration of the straight wing.

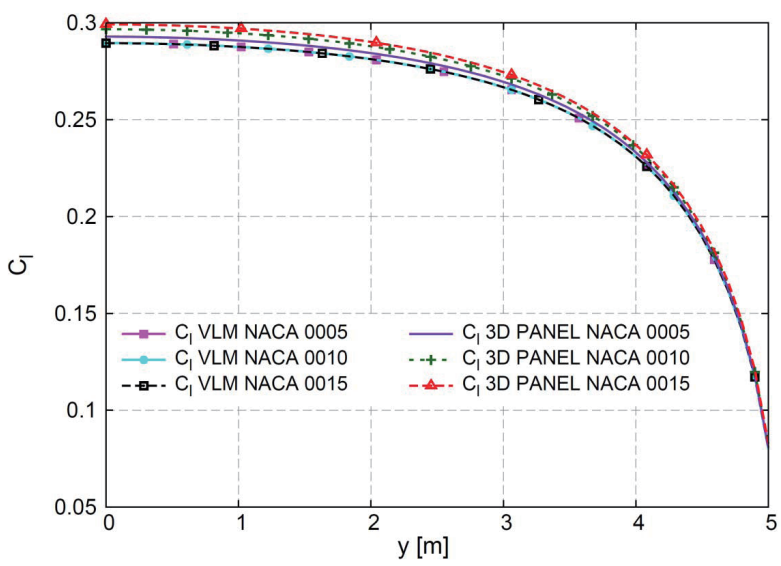

(a) Effect of the airfoil thickness

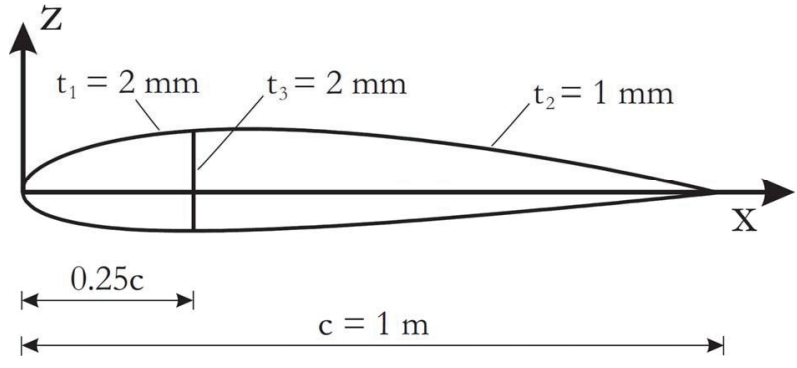

(b) NACA 2415 airfoil cross-section, with variable thickness and 2 cells

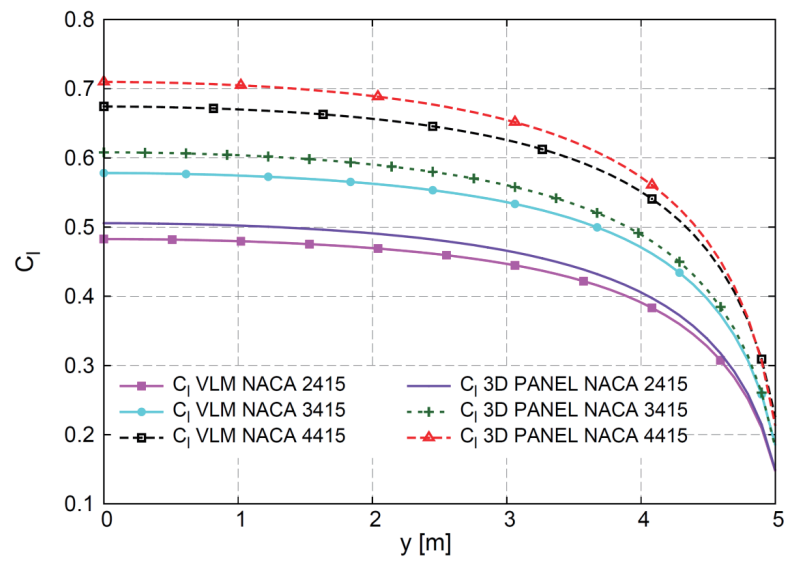

(b) Effect of the airfoil camber line

Fig. 3. Effects of the (a) airfoil thickness and (b) camber line on the spanwise local lifting coefficient $C_{1}$ of the straight wing, along the $y$ axis. Comparison of the VLM and the 3D Panel Method. $V_{\infty}=50 \mathrm{~m} / \mathrm{s}, \rho_{\infty}=1.225 \mathrm{~kg} / \mathrm{m}^{3}, \alpha=3^{\circ}$. 
aerodynamic pressures on the wing reference surface, and underestimates $C_{l}$ with respect to the 3D Panel Method. In contrast, the 3D Panel Method is able to evaluate the change of the lifting coefficient as the airfoil thickness increases, as can be seen in Fig. 3a.

Figure $3 \mathrm{~b}$ reports the trend of the spanwise local lifting coefficient $C_{l}$ as the camber line changes. It is evident that both aerodynamic methods are able to analyze the influence of the camber line. Comparing Figs. $3 a$ and $3 b$, it should be noted that the spanwise local lifting coefficient, and thus the aerodynamic pressures, are affected more by the camber line change than the airfoil thickness change. It can be concluded that the 3D Panel Method is able to provide a more realistic evaluation of the pressure distribution on the wing than the VLM. Moreover, the 3D Panel Method affords pressure loads on the actual wing surface, which are fundamental for an accurate study of the actual wing deformation and airfoil distortion, in lieu of loads applied on a fictitious wing reference surface, as for the VLM case. These reasons make the 3D Panel Method the recommended classical aerodynamic tool for the following aeroelastic wing analyses.

\subsection{Structural assessment}

In order to validate the results given by the proposed higher-order 1D CUF approach, a comparison of the static structural wing response is here performed, with MSC Nastran. Only the right half-wing of the straight configuration introduced in the previous aerodynamic assessment (see Fig. $2 a$ ) is considered here, due to loads and structural symmetry. A clamped boundary condition is taken into account for the

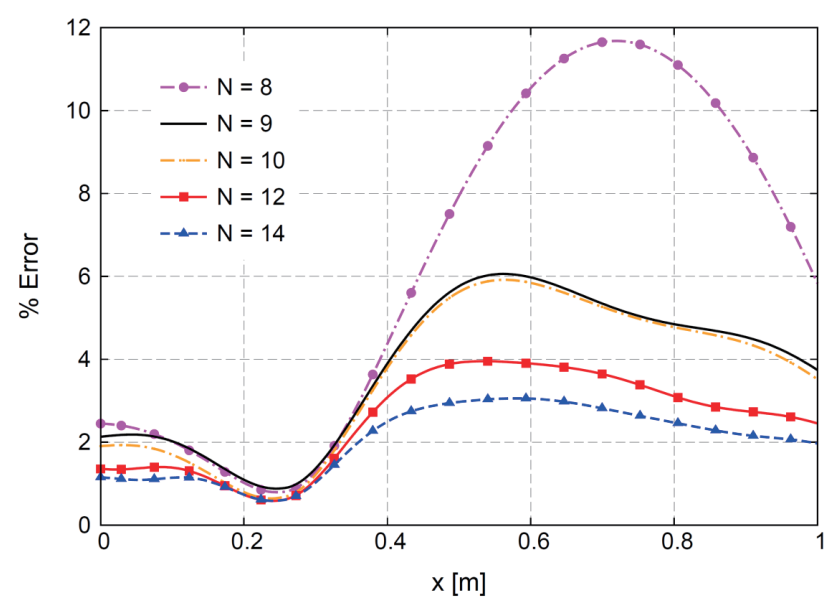

(a) Airfoil upper surface root cross-section (at $y=0$ ), whereas the tip cross-section is free. The cross-section employed is a 2415 NACA airfoil, with constant thickness equal to $2 \mathrm{~mm}$. A spar with a thickness equal to $2 \mathrm{~mm}$ is inserted along the spanwise direction, at $25 \%$ of the chord. The isotropic material adopted is aluminum: Young's modulus $E=69 G P a$, and Poisson's ratio $v=0.33$.

Due to the small thickness and the well-known aspect ratio restrictions typical of solid elements, this wing is modeled in MSC Nastran by 214,500 solid Hex8 elements and 426,852 nodes, corresponding to $1,280,556$ degrees of freedom (DOFs). The same structure is analyzed through CUF models with a variable expansion order up to $N=14$, and discretized through a 1D mesh of 10 B4 finite elements (31 nodes). The number of DOFs depends on $N$, as expressed in Eq. 2; for instance, with $10 \mathrm{~B} 4$ elements and $N=14$, the DOFs are 11,160 . However, an analysis of the present structure is also carried out through a Nastran shell FE model, but it is not reported herein, for the sake of brevity. Nonetheless, the error obtained between ID CUF and shell results is comparable with the error obtained between 1D CUF and

Table 1. Pressure distribution on the wing along the spanwise direction, for the structural assessment. $V_{\infty}=50 \mathrm{~m} / \mathrm{s}, \rho_{\infty}=1.225 \mathrm{~kg} /$ $\mathrm{m}^{3}, p_{\text {ref }}=\frac{1}{2} \rho_{\infty} V_{\infty}^{2}=551.25 \mathrm{~Pa}$.

\begin{tabular}{ll}
\hline \hline$y[\mathrm{~m}]$ & $p / p_{\text {ref }}$ \\
\hline \hline $0.00 \leq y \leq 1.25$ & 1.00 \\
$1.25<y \leq 2.50$ & 0.75 \\
$2.50<y \leq 3.75$ & 0.50 \\
$3.75<y \leq 5.00$ & 0.25 \\
\hline
\end{tabular}

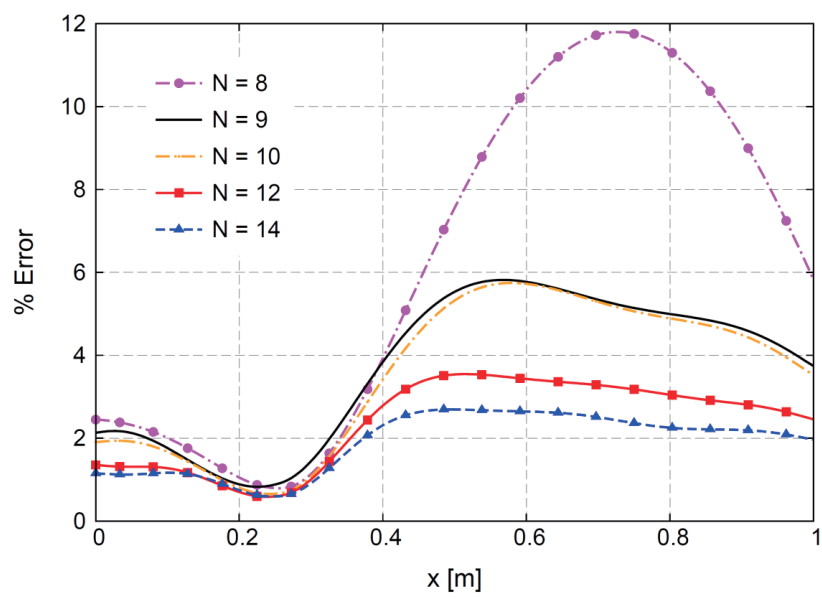

(b) Airfoil lower surface

Fig. 4. Percent error obtained by different 1D CUF models in the computation of the distortion along the airfoil (a) upper and (b) lower surfaces, at the wing tip cross-section $(y=5 m)$. Structural assessment: static wing response to a variable pressure distribution. Reference solution: Nastran solid. 
solid results.

A variable pressure distribution step-like along the spanwise direction is applied to the upper and lower wing surfaces, in order to simulate a real pressure distribution, see Table 1. The static structural response of the wing is evaluated in terms of the distortion $s$ at the tip cross-section. For the upper and lower surfaces, Figs. $4 \mathrm{a}$ and $4 \mathrm{~b}$ show the percent error $e$ obtained by computing the distortion through 1D CUF models and the Nastran solid model, which is taken as reference:

$$
e=100 \frac{S_{\text {Nastran }}-s_{1 D C U F}}{s_{\text {Nastran }}}
$$

As depicted in Figs. 4a and 4b, the proposed 1D FEs provide a convergent solution, by gradually approaching the Nastran solid results, as the expansion order increases from 8 to 14, according to the conclusions made in previous CUF works [39]. For $N=14$, the maximum percent error is about $3 \%$ for the upper surface, and about $2.7 \%$ for the lower surface. For the wing configuration considered, the choice of $N=14$ seems hence to be accurate enough to detect the crosssection distortion with an acceptable error with respect to the Nastran 3D results, and with a remarkable reduction in terms of DOFs (about a 91\% reduction, 11,160 vs. 1,280,556).

\subsection{Aeroelastic coupling}

This section focuses on the results regarding the equilibrium aeroelastic response of a wing exposed to a free stream velocity $V_{\infty}=30 \mathrm{~m} / \mathrm{s}$, via the iterative CUF-XFLR5 procedure. This analysis aims at evaluating the influence of the CUF expansion order $N$ on the aeroelastic behavior of the structure, as the accurate description of the cross-section distortion depends on $N$. The same material and straight wing configuration as those considered in the previous

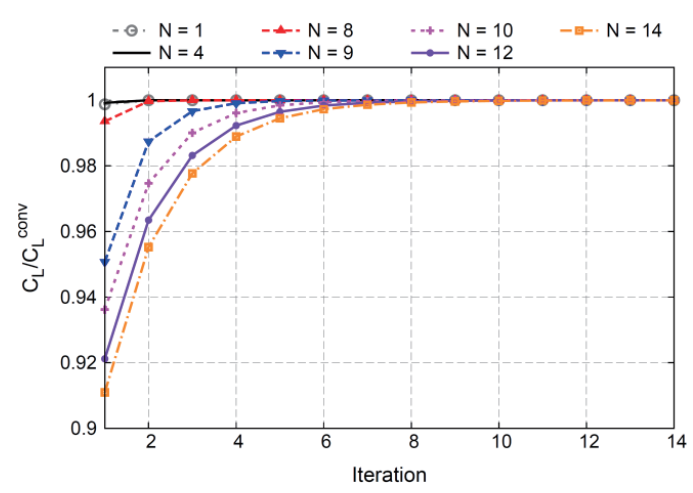

(a) Relative lifting coefficient assessment are employed here, see Fig. 2a. In this case, the cross-section is the NACA 2415 airfoil, depicted in Fig. 2b. The spar thickness $t_{3}$ is constant and equal to $2 \mathrm{~mm}$; whereas, the skin thickness of upper and lower surfaces varies gradually, from $2 \mathrm{~mm}$ ( $t_{1}$ in Fig. $\left.2 \mathrm{~b}\right)$ to $1 \mathrm{~mm}\left(t_{2}\right.$ in Fig. 2b), in the zone between $40 \%$ and $45 \%$ of the chord. This particular choice is coherent with the purpose of studying a highly-deformable nonclassical cross-section.

The 1D structural mesh consists of 10 B4 elements. For the sake of brevity, a convergent study on the number of mesh elements is not reported here. In fact, the choice of $10 \mathrm{~B} 4$ elements yields a good evaluation of displacements for all the points of the structure, as detailed in $[32,34]$, where a similar structural case in terms of wing configuration and applied aerodynamic loads was studied via the present structural model, and successfully assessed with a commercial FE solid model.

The aeroelastic analysis is now carried out following the iterative coupled procedure CUF-XFLR5 described in Fig. 1 , and varying $N$. The convergence process on the lifting and moment coefficients is drawn in Fig. 5a, by means of a dimensionless parameter $C_{L} / C_{L}^{c o n v}$, and in Fig. 5b,

Table 2. Convergent values of lifting coefficient $C_{L}^{\text {conv }}$ and moment coefficient $C_{M}^{c o n v}$, for different structural models. $V_{\infty}=30 \mathrm{~m} / \mathrm{s}$, $C_{L}^{i n}=0.4637, C_{M}^{i n}=-0.1629$.

\begin{tabular}{ccccc}
\hline \hline Model & $C_{L}^{\text {conv }}$ & $C_{M}^{\text {conv }}$ & $N_{\text {iter }}^{\text {conv } C_{L} C_{M}}$ & DOFs \\
\hline \hline$N=1$ & 0.4643 & -0.1633 & 2 & 279 \\
$N=4$ & 0.4641 & -0.1634 & 2 & 1,395 \\
$N=8$ & 0.4667 & -0.1659 & 3 & 4,185 \\
$N=9$ & 0.4877 & -0.1823 & 6 & 5,115 \\
$N=10$ & 0.4953 & -0.1886 & 8 & 6,138 \\
$N=12$ & 0.5034 & -0.1950 & 9 & 8,463 \\
$N=14$ & 0.5090 & -0.1994 & 10 & 11,160 \\
\hline
\end{tabular}

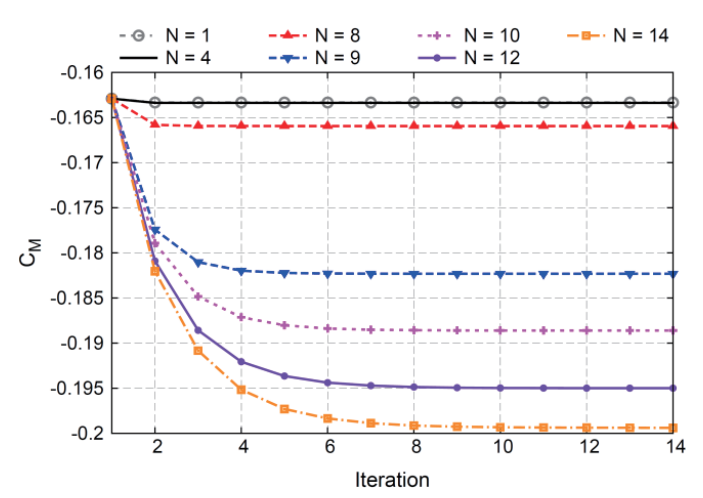

(b) Moment coefficient

Fig. 5. Convergence of lifting and moment coefficients in the iterative aeroelastic analysis, for structural models with different accuracy. Aerodynamic method: 3D Panel. $V_{\infty}=30 \mathrm{~m} / \mathrm{s}$. 
respectively. $C_{L}^{\text {conv }}$ is the final convergent value of the lifting coefficient, which is different for each expansion order employed, as well as the final convergent moment coefficient $C_{M}^{c o n v}$, as reported in Table 2.

Hence, a different choice of $N$ influences the structural response of the wing to the aerodynamic loads, and consequently also affects the aerodynamic analysis, due to the aeroelastic coupling. The higher the expansion order employed, the more difference appears between $C_{L}^{\text {conv }}$ $\left(C_{M}^{\text {conv }}\right)$ and the initial value $C_{L}^{\text {in }}\left(C_{M}^{\text {in }}\right)$ evaluated for the undeformed wing. For the cases presented in this work, the number of iterations required to achieve the convergence of the lifting coefficient is the same as that required to achieve the convergence of the moment coefficient. It can be seen that the increase of $N$ corresponds to the increasing number of iterations $N_{i t e r}^{\text {conv }} C_{L} C_{M}$ required to achieve the convergence of aerodynamic coefficients. This tendency will be clearly explained afterwards, as a consequence of the introduction of higher-order terms in the model formulation, which enriches the displacement field.

An average cross-section distortion $\bar{s}$ is now introduced in order to evaluate the aeroelastic deformation of the cross-

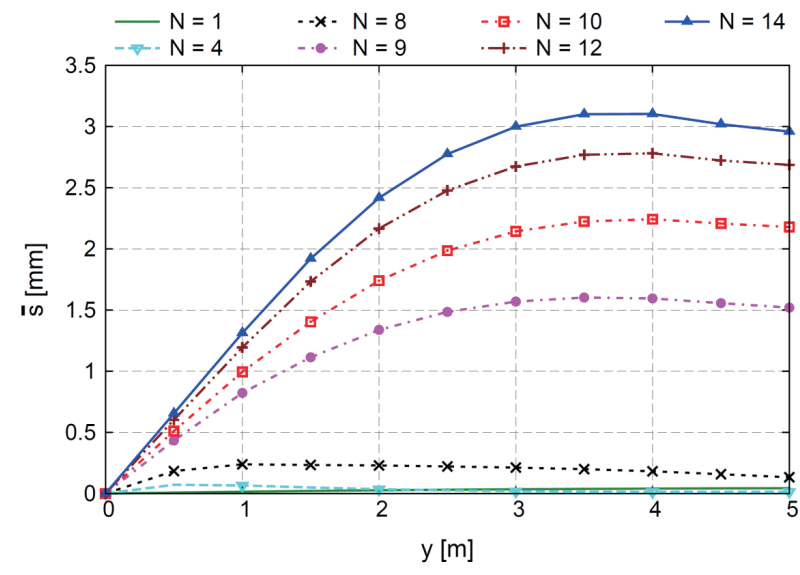

Fig. 6. Spanwise distribution of the average distortion $\bar{s}$ of the airfoil cross-sections, for different structural models. $V_{\infty}=30 \mathrm{~m} / \mathrm{s}$. section shape along the wing span. Given an airfoil crosssection, the average distortion $\bar{s}$ is defined as:

$$
\bar{s}=\frac{\oint s \cdot d l}{\oint d l}
$$

where $l$ is the curvilinear coordinate along the external airfoil surface, and $s$ is the distortion of the single point of the external airfoil surface defined in Eq. 12. It is noteworthy that $s$ is a positive quantity, and a null value for the average distortion $\bar{s}$ means no distortion. Figure 6 plots the trend of the average distortion along the wing span, showing which are the most in-plane deformed airfoil cross-sections in the static aeroelastic equilibrium response. A remarkable variation in the trend of the average distortion appears, depending on the accuracy of the structural model chosen. Models with an expansion order higher than 9 reveal that the section at $y=4 \mathrm{~m}$ appears to be the most distorted section.

For this cross-section, Table 3 presents the numerical values of average distortion $\bar{s}$ in the iterative aeroelastic analysis, for different structural theories. As occurred for the convergence of aerodynamic coefficients, the number

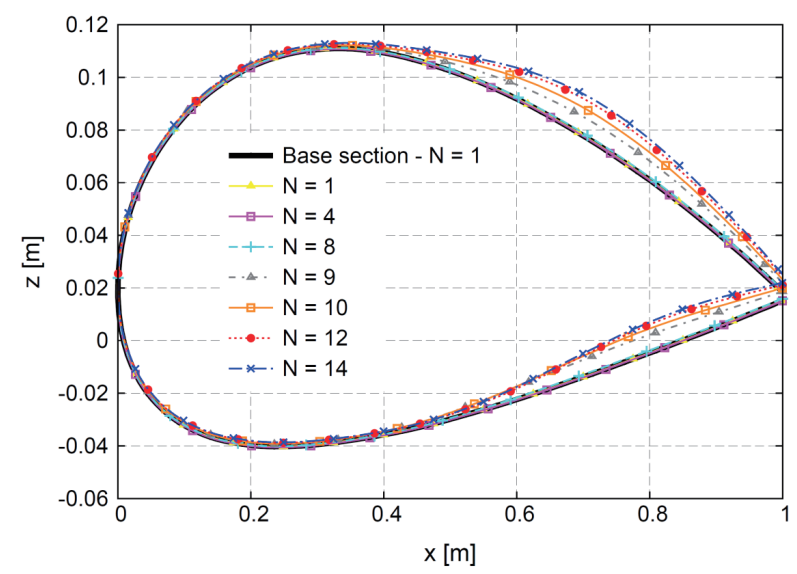

Fig. 7. Deformation of the airfoil cross-section at $y=4 \mathrm{~m}$, computed for structural models with different accuracy. $V_{\infty}=30 \mathrm{~m} / \mathrm{s}$.

Table 3. Convergence of the average distortion $\bar{s}[\mathrm{~mm}]$ in the iterative aeroelastic analysis, for different structural models. Airfoil cross-section at $y=4 \mathrm{~m} . V_{\infty}=30 \mathrm{~m} / \mathrm{s}$.

\begin{tabular}{|c|c|c|c|c|c|c|c|c|c|c|c|c|c|}
\hline \multirow{2}{*}{ Model } & \multicolumn{13}{|c|}{ Iteration } \\
\hline & 1 & 2 & 3 & 4 & 5 & 6 & 7 & 8 & 9 & 10 & 11 & 12 & 13 \\
\hline $\bar{N} N=1$ & 0.0402 & 0.0403 & 0.0403 & - & - & - & - & - & - & - & - & - & 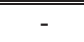 \\
\hline$N=4$ & 0.0135 & 0.0136 & 0.0136 & - & - & - & - & - & - & - & - & - & - \\
\hline$N=8$ & 0.1729 & 0.1816 & 0.1820 & 0.1821 & 0.1821 & - & - & - & - & - & - & - & - \\
\hline$N=9$ & 1.1441 & 1.4721 & 1.5624 & 1.5868 & 1.5934 & 1.5951 & 1.5956 & 1.5958 & - & - & - & - & - \\
\hline$N=10$ & 1.4177 & 1.9198 & 2.1159 & 2.1930 & 2.2234 & 2.2353 & 2.2400 & 2.2419 & 2.2426 & 2.2429 & - & - & - \\
\hline$N=12$ & 1.6738 & 2.2852 & 2.5542 & 2.6774 & 2.7340 & 2.7600 & 2.7719 & 2.7774 & 2.7799 & 2.7811 & 2.7816 & 2.7818 & - \\
\hline$N=14$ & 1.7925 & 2.4670 & 2.7867 & 2.9456 & 3.0250 & 3.0646 & 3.0844 & 3.0941 & 3.0990 & 3.1014 & 3.1027 & 3.1033 & 3.1035 \\
\hline
\end{tabular}


of iterations $N_{i t e r}^{\text {convs }}$ required to achieve the convergence of $\bar{s}$ increases as $N$, and consequently DOFs, increase. In fact, increasing the expansion order $N$, the structural model becomes, in general, more deformable, approaching the real structural behavior. This means that a complete threedimensional displacement field, as well as local effects, are evaluated with increasing accuracy, especially for structures with highly-deformable cross-sections, see Figs. $4 \mathrm{a}$ and $4 \mathrm{~b}$. Since the model accuracy increases, the structural deformation is therefore more sensitive to the variations of aerodynamic loads, which are different for each iteration, following the convergent trend in Figs. 5a and 5b, leading to an increasing $N_{i t e r}^{\text {convs }}$. The numerical results in Table 3 highlight that, given an expansion order, a higher number of iterations is necessary to achieve convergence on structural distortion than convergence on aerodynamic coefficients $\left(N_{\text {iter }}^{\text {conv }}>N_{\text {iter }}^{\text {conv } C_{L} C_{M}}\right)$, although the tolerance employed is

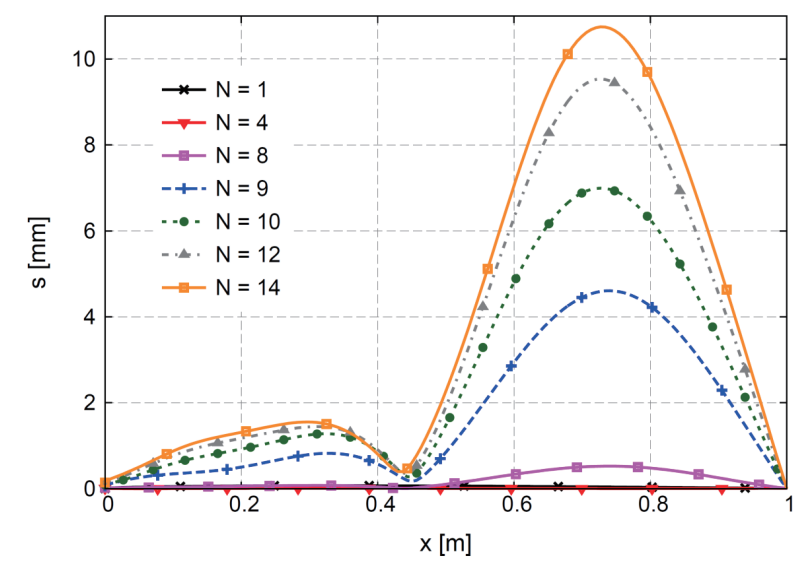

Fig. 8. Distortion of the airfoil upper surface of the cross-section at $y=4 \mathrm{~m}$, computed for different structural models. $V_{\infty}=30 \mathrm{~m} / \mathrm{s}$.

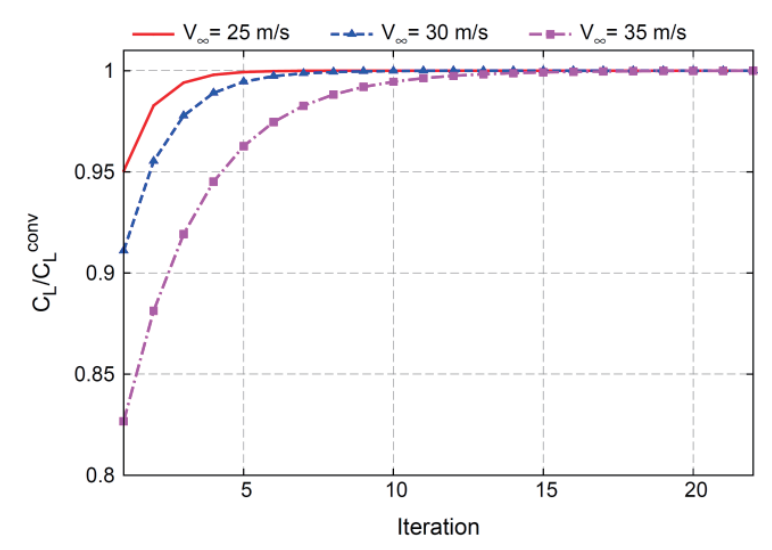

(a) Relative lifting coefficient the same.

For $N>8$, the displacement field becomes accurate enough to relevantly take into account a cross-section distortion for the airfoil case considered, as can also be seen in Fig. 7. As previously explained, given a structural model, the distortion is computed by comparing the deformed cross-section to the corresponding base section. For the sake of simplicity, only the base section for $N=1$ is plotted in Fig. 7 .

As expected, low-order models provide a correct evaluation of the bending and torsional structural behavior, but not an exhaustive description of the in-plane deformation. This conclusion is confirmed by Fig. 8, where the airfoil distortion $s$ computed by variable kinematic models is depicted along the upper surface, at $y=4 \mathrm{~m}$. The maximum distortion value is reached in the part of the cross-section next to the trailing edge, since the stiffening effect due to the spar at $25 \%$ of the chord limits the crosssection distortion. Nonetheless, the chordwise position of the maximum distortion points on the airfoil upper and lower surfaces changes, depending on the accuracy of the structural model, see Table 4. As a consequence, it is worth pointing out that the increase of $N$ is relevant, not only for an accurate detection of distortion values, but also of the accurate shape-type deformation.

In general, improvements of the structural theory yield more realistic deformations of the wing, until a good convergence is achieved for $N=14$, according to the conclusions made for Figs. $4 \mathrm{a}$ and $4 \mathrm{~b}$ in the structural assessment. In other words, the difference between the results obtained through the generic $(N-1)$-th and $N$-th expansion orders decreases and becomes minimal for $N=14$. For this reason, it is possible to consider the fourteenthorder model sufficiently accurate to describe the aeroelastic

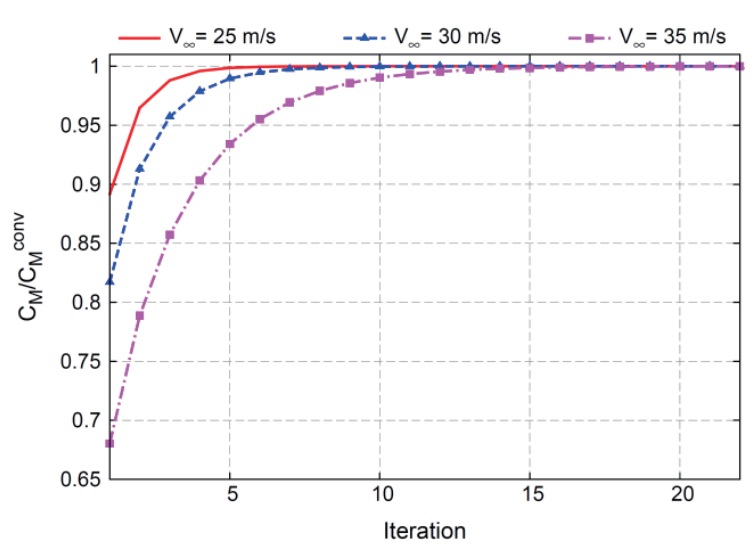

(b) Relative moment coefficient

Fig. 9. Convergence of lifting and moment coefficients in the iterative aeroelastic analysis, for different free stream velocities. Structural model: $N$ $=14$. Aerodynamic method: $3 \mathrm{D}$ Panel. 
behavior of the structure here considered.

\subsection{Free stream velocity influence}

This analysis aims at establishing the influence of the free stream velocity on the wing distortion. The wing configuration employed for this analysis is the same as that used in the previous study. According to the previous conclusion,

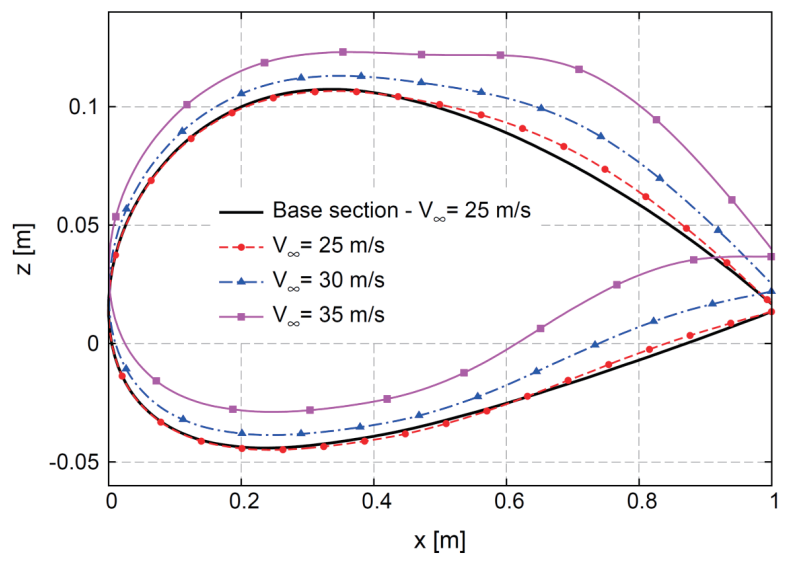

Fig. 10. Deformation of the airfoil cross-section at $y=4 \mathrm{~m}$, computed for different free stream velocities. Structural model: $N=14$. the structural model considered is $N=14$. The free stream velocities considered are 25,30 , and $35 \mathrm{~m} / \mathrm{s}$. As in the previous analysis, the aerodynamic convergence process is presented through the dimensionless parameter $C_{L} / C_{L}^{\text {conv }}$, as illustrated in Fig. 9a. The convergence of the moment coefficient is also shown in Fig. 9b, through the parameter $C_{M} / C_{M}^{\text {conv }}$.

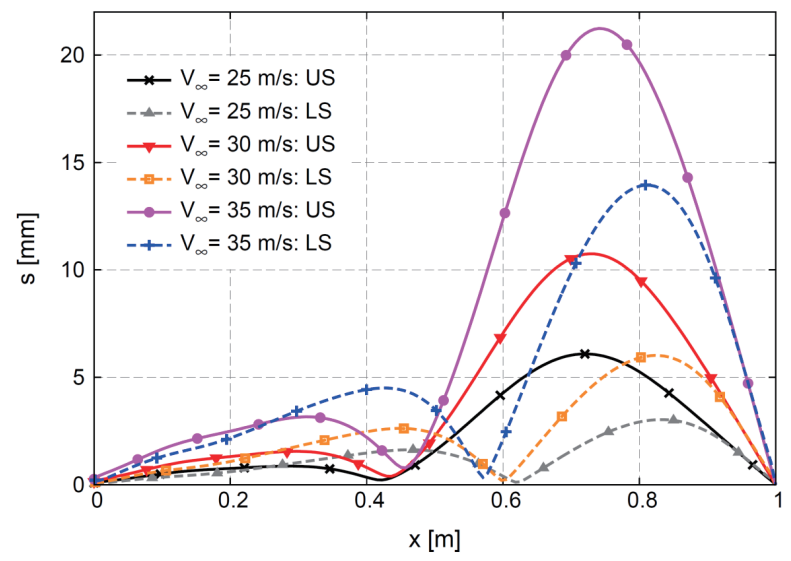

Fig. 11. Distortion of the airfoil upper and lower surfaces of the crosssection at $y=4 m$, computed for different free stream velocities. Structural model: $N=14$.

Table 4. Convergent average distortion $\bar{s}^{\text {conv }}[\mathrm{mm}]$ of the cross-section at $\mathrm{y}=4 \mathrm{~m}$, for different structural models. Values and chordwise positions of the maximum distortions $s_{\max }^{U S}[\mathrm{~mm}]$ and $s_{\max }^{L S}[\mathrm{~mm}]$, on the airfoil upper and lower surfaces. $V_{\infty}=30 \mathrm{~m} / \mathrm{s}$.

\begin{tabular}{cccccccc}
\hline \hline Model & $\bar{s}^{\text {conv }}$ & $N_{\text {iter }}^{\text {conv }}$ & $s_{\max }^{U S}$ & $x_{s_{\max }^{U S}} / c$ & $s_{\max }^{L S}$ & $x_{s_{\max }^{L S}} / c$ & DOFs \\
\hline \hline$N=1$ & 0.0403 & 3 & 0.0718 & 0.33 & 0.0439 & 0.24 & 279 \\
$N=4$ & 0.0136 & 3 & 0.0103 & 0.33 & 0.0251 & 0.23 & 1,395 \\
$N=8$ & 0.1821 & 5 & 0.5267 & 0.74 & 0.4797 & 0.75 & 4,185 \\
$N=9$ & 1.5958 & 8 & 4.6073 & 0.74 & 4.1253 & 0.75 & 5,115 \\
$N=10$ & 2.2429 & 10 & 6.9936 & 0.73 & 5.1626 & 0.79 & 6,138 \\
$N=12$ & 2.7818 & 12 & 9.5341 & 0.73 & 5.7456 & 0.82 & 8,463 \\
$N=14$ & 3.1035 & 13 & 10.7482 & 0.73 & 6.0178 & 0.82 & 11,160 \\
\hline
\end{tabular}

Table 5. Convergent values of lifting coefficient $C_{L}^{\text {conv }}$, moment coefficient $C_{M}^{\text {conv }}$, and average distortion $\bar{s}^{\text {conv }}$ [mm] of cross-section at $\mathrm{y}=4 \mathrm{~m}$, for different free stream velocities $V_{\infty}[\mathrm{m} / \mathrm{s}]$. Structural model: $N=14 . C_{L}^{i n}=0.4637, C_{M}^{i n}=-0.1629$.

\begin{tabular}{cccccc}
\hline \hline$V_{\infty}$ & $C_{L}^{\text {conv }}$ & $C_{M}^{\text {conv }}$ & $N_{\text {iter }}^{\text {conv } C_{L} C_{M}}$ & $\bar{S}^{\text {conv }}$ & $N_{\text {iter }}^{\text {cons }}$ \\
\hline \hline 25 & 0.4879 & -0.1827 & 7 & 1.7269 & 9 \\
30 & 0.5090 & -0.1994 & 10 & 3.1035 & 13 \\
35 & 0.5608 & -0.2394 & 18 & 6.3296 & 22 \\
\hline
\end{tabular}

Table 6. Values and chordwise positions of the maximum distortions $s_{\max 1}^{U S}, s_{\max 2}^{U S}, s_{\max 1}^{L S}, s_{\max 2}^{L S}[\mathrm{~mm}]$ on the airfoil upper and lower surfaces of the cross-section at $y=4 \mathrm{~m}$, for different free stream velocities $V_{\infty}[\mathrm{m} / \mathrm{s}]$. Structural model: $N=14$.

\begin{tabular}{ccccccccc}
\hline \hline$V_{\infty}$ & $s_{\max 1}^{U S}$ & $x_{s_{\max 1}^{U S}} / c$ & $s_{\max 2}^{U S}$ & $x_{s_{\max 2}^{U S}} / c$ & $s_{\max 1}^{L S}$ & $x_{s_{\max 1}^{L S}} / c$ & $s_{\max 2}^{L S}$ & $x_{s_{\max 2}^{L S}} / c$ \\
\hline \hline 25 & 6.0892 & 0.72 & 0.8670 & 0.29 & 3.0324 & 0.83 & 1.6285 & 0.46 \\
30 & 10.7482 & 0.73 & 1.5540 & 0.30 & 6.0178 & 0.82 & 2.6232 & 0.45 \\
35 & 21.2323 & 0.74 & 3.1618 & 0.31 & 13.9437 & 0.81 & 4.5026 & 0.43 \\
\hline
\end{tabular}


In this case, $C_{L}^{c o n v}$ and $C_{M}^{c o n v}$ represent the final convergent values of the lifting and moment coefficients for a given $V_{\infty}$, respectively. As occurred for the previous aeroelastic analysis, the trends do not show any numerical problems, such as oscillations. From Figs. 9a and 9b, it is important to note that the number of iterations $N_{i t e r}^{\text {conv }} C_{L} C_{M}$ required to achieve the aerodynamic convergence increases as $V_{\infty}$ increases, and the final convergent values are much different from the initial values, as summarized in Table 4 . The reason for this behavior is easily explained by the fact that an increasing free stream velocity means increasing aerodynamic loads, and consequently higher structural deformations, and lastly, a more relevant coupling effect on the aeroelastic response of the wing. In fact, an increasing airfoil distortion for the most deformed cross-section at $y=4$ m is obtained with $V_{\infty}$, according to the numerical results in Table 5 and airfoil deformed profiles in Fig. 10. Also for velocity values different from $30 \mathrm{~m} / \mathrm{s}$, a higher number of iterations is necessary to achieve convergence on structural distortion than convergence on the aerodynamic lifting coefficient $\left(N_{\text {iter }}^{\text {convs }}>N_{\text {iter }}^{\text {conv } C_{L} C_{M}}\right)$, see Table 5 .

The limitation of distortion close to the airfoil leading edge, due to the spar, is enhanced for $V_{\infty}=35 \mathrm{~m} / \mathrm{s}$. The trends of distortion on the airfoil upper and lower surfaces, which are indicated as US and LS, respectively, are depicted in Fig. 11 at $y=4$, for different velocities. It is important to note that deformations of the upper and lower surfaces also remarkably differ, because of different aerodynamic pressure distributions. Table 6 shows that not only the maximum distortion values on the airfoil upper $\left(s_{\max 1}^{U S}, s_{\max 2}^{U S}\right)$ and lower $\left(s_{\max 1}^{L S}, s_{\max 2}^{L S}\right)$ surfaces changes as $V_{\infty}$ varies, but also their corresponding chordwise positions. This aspect highlights the importance of higher-order models, in particular for an accurate evaluation of the in-plane crosssection distortion of highly-deformable structures.

\section{Conclusions}

Variable kinematic 1D finite elements were formulated on the basis of the Carrera Unified Formulation (CUF) and coupled to an aerodynamic 3D panel method, implemented in XFLR5. The aeroelastic static response of a straight wing with a highly-deformable airfoil cross-section was computed through a coupled iterative procedure, for increasing structural accuracy and for different free stream velocities. An aerodynamic assessment confirmed that the 3D Panel Method provides a more realistic evaluation of the pressure distribution on the wing, than the Vortex Lattice Method
(VLM). As far as the use of 1D higher-order models is concerned, the following main conclusions can be drawn:

1. The introduction of higher-order terms in the displacement field is even more important for the aeroelastic analysis, due to the fluid-structure coupling.

2. In the case that the wing is rather flexible, the in-plane cross-section deformation has a great impact on the alteration of the aerodynamic loadings.

3. The higher the free stream velocity, the more marked the in-plane distortion effect.

As far as the present hierarchical one-dimensional approach is concerned, the results point out that:

a. The CUF is an ideal tool to easily compare different higher-order theories, since the model accuracy is a free parameter of the analysis.

b. The in-plane airfoil cross-section deformation is welldescribed by the proposed 1D structural model, in good agreement with a three-dimensional FE solution, and with a remarkable reduction in terms of DOFs.

c. A convergent trend of displacements and aerodynamic coefficients is achieved as the structural model accuracy increases. This proves that the proposed 1D higher-order approach does not introduce additional numerical problems in the aeroelastic analysis of wings with arbitrary cross-section geometry.

d. A higher number of iterations is necessary to achieve convergence on structural distortion than for convergence on aerodynamic coefficients.

These reasons make the future use of the proposed CUFXFLR5 approach appear promising for a versatile flight optimization tool.

\section{References}

[1] Fung, Y.C., An Introduction to the Theory of Aeroelasticity, Dover Publications, 2008.

[2] Patil, M.J., Hodges D.H., and Cesnik C.E.S., "Nonlinear aeroelasticity and flight dynamics of high-altitude longendurance aircraft", Journal of Aircraft, Vol. 38, No. 1, 2001, pp. 88-94.

[3] Sulaeman, E., Kapania, R., and Haftka, R.T., "Parametric studies of flutter speed in a strut-braced wing", Proceeding of the 43rd AIAA/ASME/ASCE/AHS/ASC Structures, Structural Dynamics, and Materials Conference, AIAA Paper 2002-1487, Denver, CO, April 2002.

[4] Hodges, D.H., and Pierce, G.A., Introduction to Structural Dynamics and Aeroelasticity, Cambridge University Press, 2002. 
[5] Sofla, A.Y.N., Meguid, S.A., Tan, K.T., and Yeo, W.K., "Shape morphing of aircraft wing: status and challenges", Materials \& Design, Vol. 31, No. 3, 2010, pp.1284-1292.

[6] Gamboa, P., Vale, J., Lau, F.J.P., and Suleman, A., "Optimization of a morphing wing based on coupled aerodynamic and structural constraints", AIAA Journal, Vol. 47, No. 9, 2009, pp. 2087-2104.

[7] Suleman, A., and Costa. A.P., "Adaptive control of an aeroelastic flight vehicle using piezoelectric actuators", Computers \& Structures, Vol. 82, No. 17-19, 2004, pp. 13031314.

[8] Kota, S., Hetrick, J.A., Osborn, R., Paul, D., Pendleton, E., Flick, P., and Tilmann, C., "Design and application of compliant mechanisms for morphing aircraft structures", Proceedings of the SPIE Vol. 5054, Smart Structures and Materials 2003: Industrial and Commercial Applications of Smart Structures Technologies, San Diego, CA, 12 August 2003.

[9] Diaconu, C.G., Weaver, P.M., and Mattioni, F., "Concepts for morphing airfoil sections using bi-stable laminated composite structures", Thin-Walled Structures, Vol. 46, No. 6, 2008, pp. 689-701.

[10] Lim, S.M., Lee, S., Park, H.C., Yoon, K.J. and Goo, N.S., "Design and demonstration of a biomimetic wing section using a lightweight piezo-composite actuator (LIPCA)", Smart Materials and Structures, Vol. 14, No. 4, 2005, pp. 496503.

[11] Elzey, D.M., Sofla, A.Y.N., and Wadley, H.N.G., "A shape memory-based multifunctional structural actuator panel", International Journal of Solids and Structures, Vol. 42, No. 7, 2005, pp. 1943-1955.

[12] Bhardwaj, M.K., "Aeroelastic analysis of modern complex wings using ENSAERO and NASTRAN", Technical Report NASA 19980235582, 1995.

[13] Dowell, E.H., and Hall, K.C., "Modeling of fluidstructure interaction", Annual Review of Fluid Mechanics, Vol. 33, 2001, pp. 445-490.

[14] Henshaw, M.J. de C. et al., "Non-linear aeroelastic prediction for aircraft applications", Progress in Aerospace Sciences, Vol. 43, No. 4-6, 2007, pp. 65-137.

[15] Katz, J., and Plotkin, A., Low-Speed Aerodynamics, Cambridge University Press, 2001.

[16] Wang, Y., Wan, Z., and Yang, C., "Application of highorder panel method in static aeroelastic analysis of aircraft", Procedia Engineering, Vol. 31, 2012, pp. 136-144.

[17] Yang, C., Zhang, B.C., Wan, Z.Q., and Wang, Y.K., "A method for static aeroelastic analysis based on the highorder panel method and modal method", Science China Technological Sciences, Vol. 54, No. 3, 2011, pp. 741-748.

[18] Newman, J.C. III, Newman, P.A., Taylor, A.C. III, and
Hou, G.J.W., "Efficient nonlinear static aeroelastic wing analysis", Computers \& Fluids, Vol. 28, No. 4, 1999, pp. 615628.

[19] Bathe, K.J., Finite Element Procedures, Prentice Hall, Upper Saddle River, New Jersey, 1996.

[20] Kapania, K., and Raciti, S, "Recent advances in analysis of laminated beams and plates, part II: Vibrations and wave propagation", AIAA Journal, Vol. 27, No. 7, 1989, pp. 935-946.

[21] El Fatmi, R., "Non-uniform warping including the effects of torsion and shear forces. Part I: A general beam theory", International Journal of Solids and Structures, Vol. 44, No. 18-19, 2007, pp. 5912-5929.

[22] Schardt, R., "Extension of the engineer's theory of bending to the analysis of folded plate structures", Der Stahlbau, Vol. 35, 1966, pp. 161-171.

[23] Silvestre, N., and Camotim, D., "Second-order generalised beam theory for arbitrary orthotropic materials", Thin-Walled Structures, Vol. 40, No. 9, 2002, pp. 791-820.

[24] Berdichevsky, V.L., Armanios, E., and Badir, A., "Theory of anisotropic thin-walled closed-cross-section beams", Composites Engineering, Vol. 2, No. 5-7, 1992, pp. 411-432.

[25] Yu, W., Volovoi, V.V., Hodges, D.H., and Hong, X., "Validation of the variational asymptotic beam sectional analysis (VABS)", AIAA Journal, Vol. 40, No. 10, 2002, pp. 2105-2113.

[26] Patil, M.J., "Aeroelastic tailoring of composite box beams", Proceedings of the 35th Aerospace Sciences Meeting and Exhibit, AIAA Paper 97-0015, Reno, NV, January 1997.

[27] Librescu, L., and Song., O., “On the static aeroelastic tailoring of composite aircraft swept wings modelled as thinwalled beam structures", Composites Engineering, Vol. 2, No. 5-7, 1992, pp. 497-512.

[28] Qin, Z., and Librescu, L., "Aeroelastic instability of aircraft wings modelled as anisotropic composite thinwalled beams in incompressible flow", Journal of Fluids and Structures, Vol. 18, No. 1, 2003, pp. 43-61.

[29] Jeon, S.M., and Lee, I., "Aeroelastic response and stability analysis of composite rotor blades in forward flight", Composites: Part B, Vol. 32, No. 1, 2001, pp. 249-257.

[30] Friedmann, P.P., Glaz, B., and Palacios, R., "A moderate deflection composite helicopter rotor blade model with an improved cross-sectional analysis", International Journal of Solids and Structures, Vol. 46, No. 10, 2009, pp. 2186-2200.

[31] Carrera, E., Giunta, G., and Petrolo, M., Beam Structures: Classical and Advanced Theories, John Wiley \& Sons, 2011.

[32] Varello, A., Carrera, E., and Demasi, L., "Vortex lattice method coupled with advanced one-dimensional structural 
models", Journal of Aeroelasticity and Structural Dynamics, Vol. 2, No. 2, 2011, pp. 53-78.

[33] Varello, A., Demasi, L., Carrera, E., and Giunta, G., "An improved beam formulation for aeroelastic applications", Proceedings of the 51st AIAA/ASME/ASCE/AHS/ASC Structures, Structural Dynamics, and Materials Conference, AIAA Paper 2010-3032, Orlando, FL, 12-15, April 2010.

[34] Carrera, E., Varello, A., and Demasi, L., "A refined structural model for static aeroelastic response and divergence of metallic and composite wings", CEAS Aeronautical Journal, Vol. 4, No. 2, 2013, pp. 175-189.

[35] Petrolo, M., "Flutter analysis of composite lifting surfaces by the 1D Carrera Unified Formulation and the doublet lattice method", Composite Structures, Vol. 95, 2013, pp. 539-546.

[36] Maskew, B., "Program VSAERO theory Document: a computer program for calculating nonlinear aerodynamic characteristics of arbitrary configurations", NASA contractor report CR-4023, 1987.

[37] Oosthuizen, P.H., and Carscallen, W.E., Compressible fluid flow, McGraw-Hill, 1997.

[38] Deperrois, A., Guidelines for XFLR5 v6.03, 2011.

[39] Carrera, E., and Varello, A., "Dynamic response of thin-walled structures by variable kinematic onedimensional models", Journal of Sound and Vibration, Vol. 331, No. 24, 2012, pp. 5268-5282. 